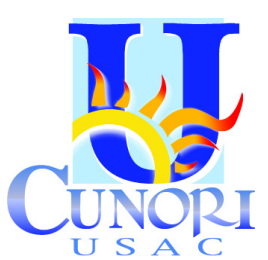

\title{
Revista Ciencia Multidisciplinaria CUNORI. \\ Competencias del profesor de la carrera de Administración de Empresas del Centro Universitario de Zacapa -CUNZAC-
}

\section{Competences of the professor of the Business Administration career of the Zacapa University Center -CUNZAC-}

\author{
Marco Antonio Rosales Gómez \\ Universidad de San Carlos de Guatemala \\ https://orcid.org/0000-0002-1728-3962 \\ ing.rosales.usac@gmail.com
}

Recibido: 25/10/2020

Publicado: 19/01/2021

\author{
Referencia del artículo \\ Rosales Gómez, M. A. (2021). Competencias del profesor de \\ la carrera de Administración de Empresas del Centro Universi- \\ tario de Zacapa -CUNZAC-. Revista Ciencia Multidisciplinaria \\ CUNORI. 5(1). 51-64. \\ DOI: https://doi.org/10.36314/cunori.v5i1.152
}

.




\section{Abstract}

as universidades están experimentando cambios profundos en la búsqueda - de la calidad del aprendizaje, que permita formar profesionales responsables, competentes y comprometidos que la sociedad actual necesita; pero esto, solo es posible desde una nueva concepción del profesor en donde debe adoptar un rol de facilitador. Para esto, es fundamental conocer las competencias que posee e identificar las que debe desarrollar para mejorar la práctica en entornos de aprendizaje con metodologías activas y participativas. Dentro del Centro Universitario de Zacapa -CUNZAC-, por ser un centro de reciente creación (año 2011), cuenta con carreras que aún no han cumplido un ciclo de cierre de pensum, lo que permite deducir que los profesores de la carrera, son también de reciente incorporación al curso que imparten, esto se convierte en un principal fundamento para que previo a invertir esfuerzos en la formación de sus profesores, se determine las competencias que poseen, lo cual será el punto de inicio para contar con profesores que trabajan por competencias y por ende los estudiantes son abordados bajo un proceso educativo participativo, activo e innovador.

\section{Keywords}

Competences, university teaching, research and innovation, connection with society, institutional management, values and attitudes of the personal and interpersonal sphere, transverse competences, specific competences 


\section{Introducción}

Desde el ámbito de las competencias en el docente del nivel superior, según Torres, Badillo, Valentín y Ramírez (2014) abarca todo lo relacionado con la práctica, finalidad y profesionalización docente. Por ello, para conocerlas es necesario considerar tres elementos: el contenido, la clasificación y la formación; es decir, saber enseñar, cómo enseñar, a quiénes se enseña y para qué.

No existe una definición estándar entre los especialistas sobre el término competencias, pues este varía en función del ámbito en el que se aplica. Al respecto, una de las primeras definiciones sobre competencias docentes, es la ofrecida por Aylett y Gregory (1997) quienes la definieron a través de dos criterios: competencia de la función docente y criterios de excelencia. Las competencias de la función docente se entienden como el conjunto de características que se manifiestan en función de organización, presentación de la información, relaciones interpersonales, orientación y evaluación, y las competencias de excelencia, se entienden como el alto nivel que el docente debe poseer en cuanto a ser reflexivo con respecto a su propia práctica, innovador, diseñador del currículum, investigador sobre aspectos docentes, organizador de cursos y líder de grupos docentes.

Tobón (2007) y Villela (2018) la definen como: procesos complejos de desempeño, que integran diferentes saberes (saber ser, saber hacer, saber conocer y saber convivir), para realizar actividades y/o resolver problemas con sentido de reto, motivación, flexibilidad, creatividad, comprensión y emprendimiento, dentro de una perspectiva de procesamiento metacognitivo, mejoramiento continuo y compromiso ético, con la meta de contribuir en todos los ámbitos de desarrollo del ser humano.

El profesor universitario en su rol de facilitador de aprendizaje, debe reunir competencias docentes que le permita ser parte de la innovación curricular que se ha venido impulsando para la formación en la educación superior. Es por ello, que se contempla en la Universidad de San Carlos de Guatemala, una reflexión sobre el modelo educativo y la problemática que persiste para lograr la calidad educativa, en ese sentido, argumenta que el acelerado flujo de la información, la producción de conocimiento y los avances de la ciencia y la tecnología producto de la Tercera Revolución Científico Tecnológica, aunados a la transición demográfica y polarización de la sociedad guatemalteca, producto del modelo de desarrollo de los úl- 
timos sesenta años, demandan nuevos retos para que la USAC como líder de la educación superior del país, desarrolle nuevos modelos educativos sustentados en enfoques complejos en concordancia con la evolución del conocimiento y las necesidades de la sociedad.

En las últimas décadas ha cobrado interés el modelo de competencias docentes y se han proliferado los estudios sobre el tema, al respecto Torello (2012) realizó un estudio en la Universidad Autónoma de Barcelona, acerca de las competencias del docente universitario, considerando seis competencias de la función docente. De ello se obtuvo que las competencias más valoradas son aquellas que se relacionan directamente con el aula, como el diseño, tutoría y evaluación, y las menos valoradas son las que se consideran como menos esenciales a la docencia y más externos al aula. Esto confirma que la percepción de todas las partes involucradas sigue enfocándose en lo que pasa de forma directa en el aula universitaria, lugar donde se interactúa y se comparte de manera constante las experiencias y saberes tanto del profesor como de los estudiantes.

Según el estudio realizado por Villaroel y Bruna (2016) en su investigación acerca de competencias pedagógicas del docente universitario, se concluye que las competencias cognitivas, comunicativas, personales, sociales y tecnológicas tiene una mayor importancia para el estudiante y las competencias que están mas desarrollas en el docente son las orientadas a las habilidades didácticas, habilidades de planificación y evaluación. De lo anterior se muestra que el profesor interactúa con los estudiantes dentro del salón de clase, es capaz de relacionar la teoría con la práctica, tiene un rol motivador, realiza grupos de trabajo y relaciona los contenidos teóricos con la vida laboral. Como análisis general se concluye que el profesor no solo debe de dominar contenidos a los futuros profesionales, sino que también debe de esforzarse en que estos logren perfiles de egreso de alta complejidad, que incluyan procedimientos, habilidades de pensamiento de orden superior y actitudes como lo afirman (Flores, 2018; Alvarado, 2020 y Arrieta, 2020).

Dentro de un contexto nacional, Zuñiga (2018) afirma que existe coherencia entre el actuar docente y la formación del estudiante además de manifestar que la memorización no es lo más importante en el estudiante. La investigación arrojó que existe una correlación entre el conocimiento, la praxis y la actitud docente. Otro indicador relevante fue que los profesores son conscientes sobre de la importancia de la formación psicopedagógica e intercultural, que siguen teniendo la necesidad de conocer más sobre 
diversas estrategias de enseñanza, lo que conlleva un compromiso de su parte para su realización (Julián, 2018).

Todo esto ha llevado a la Universidad de San Carlos de Guatemala a una reflexión sobre el modelo educativo, la problemática que persiste para alcanzar la calidad educativa, y los nuevos retos que demandan que la USAC desarrolle nuevos modelos educativos que tengan concordancia con la evolución del conocimiento y las necesidades actuales de la sociedad. Por ello, se ha desarrollado un nuevo modelo educativo que responde a la formación por competencias profesionales (USAC, 2015). Para ello, se incorpora como uno de los elementos principales la formación docente por competencias, puesto que es el profesor el pilar fundamental para llevar a cabo un modelo educativo.

De acuerdo a ello, en el Plan Académico de Desarrollo Estratégico USAC (2014) se contempla en el eje de desarrollo del recurso humano, tres acciones estratégicas: innovar la metodología y tecnología apropiada para el desarrollo de las unidades académicas en función de la calidad y la excelencia académica, actualizar y fortalecer la carrera docente, fortalecer y desarrollar los programas para la formación y capacitación de los cuerpos docentes en función de la excelencia académica y profesional, así como, acreditar los sistemas de evaluación por competencias para docentes en la universidad.

Lo anterior refleja la instrucción dada a cada unidad académica para aplicar la evaluación por competencias para garantizar el perfil profesional deseado en el desempeño del profesor universitario. Para ello, debe conocer las competencias con las que cuentan los profesores y luego implementar programas de formación, en función de los resultados. Es esto lo que hace necesaria la investigación en el Centro Universitario de Zacapa -CUNZAC-, debido a que se sigue trabajando bajo la metodología basada en objetivos, lo que restringe en cierta forma la utilización de diferentes estrategias de enseñanza por parte del profesor universitario, se sigue trabajando para cubrir metas que no muestran la realidad con la que el estudiante debe de terminar en cada curso de la carrera.

Con base en lo anterior, se puede decir que el profesor debe transmitir al estudiante las competencias que posee en cierta área profesional de la carrera, al adquirir ese compromiso y determinación de lo que debe de hacerse para mejorar el nivel educativo, personal, de investigación, inno- 
vación, gestión, y así lograr un aprendizaje significativo y permanente lo que por consecuencia conllevará un mejor profesional formado del centro universitario.

De no diagnosticar y desarrollar las competencias del profesor, se corre el riesgo de seguir dentro de los modelos tradicionales, pudiendo quedar al margen de otras universidades que ya manejan ciertos perfiles del profesor, adaptando modelos creativos y funcionales y que concluyen en un mejor rendimiento estudiantil, con fuertes valores personales e institucionales y con estudiantes mejor preparados para la vida laboral.

Derivado de lo anterior, los resultados reflejados en la investigación servirán de punto de partida para la mejora del profesor de la carrera de Administración de Empresas, ya que se aportan datos significativos y confiables, y que además de mostrar las competencias del profesor, también servirá para que a corto plazo se cree un perfil competencial del profesor de dicha carrera.

\section{Materiales y métodos}

La investigación según su alcance es de tipo descriptiva, debido a que lo que se pretendió fue determinar las competencias del profesor de la carrera de Administración de Empresas del Centro Universitario de Zacapa. Por su temporalidad es una investigación transversal. Por tal razón también está marcado en el enfoque mixto ya que se realizaron procesos sistemáticos, empíricos y críticos de investigación e implican la recolección y el análisis de datos cuantitativos y cualitativos, así como su integración y discusión conjunta, para realizar inferencias producto de toda la información recabada (meta inferencias) y lograr un mayor entendimiento del fenómeno bajo estudio (Hernández y Mendoza, 2018).

La recolección de información se realizó a dos sujetos de estudio: se recabó la percepción de los estudiantes y también se consideró la autopercepción de profesores sujetos de estudio de la carrera de Administración de Empresas del Centro Universitario de Zacapa. En cuanto a las fuentes secundarias, se hizo uso de libros de texto, artículos de revistas científicas relación el objeto de estudio, de igual forma literatura que sustenta la metodología de la investigación utilizada. 
Para la investigación se utilizó el modelo trabajado en conjunto por Marta Ruiz Corbella y Ruth Marlene Aguilar Feijoo publicado en el año 2017, en donde señala las competencias transversales y específicas. Las competencias del profesor universitario se desglosan en 20 competencias y 114 indicadores, considerando las funciones del profesor universitario las cuales son: docencia, investigación, vinculación con la sociedad y la gestión, además de los valores y actitudes del ámbito personal e interpersonal del profesor universitario. Los resultados se realizaron bajo la perspectiva del estudiante.

\section{Resultados}

Para la investigación se consideró a los estudiantes del III, V y VII de la carrera de Administración de Empresas del -CUNZAC-, en cuanto a los estudiantes del I ciclo no se consideraron debido a la naturaleza del estudio, ya que aún está en adaptación dentro de la universidad. En la siguiente tabla se muestra la distribución de los estudiantes por ciclo que cursa.

Tabla No. 1 Distribución de estudiantes por ciclo

\begin{tabular}{cccc}
\hline Ciclos & Asignados & Encuestados & Porcentaje Alcanzado \\
\hline III & 59 & 51 & 86.4 \\
\hline $\mathrm{V}$ & 69 & 65 & 94.2 \\
\hline $\mathrm{VII}$ & 65 & 54 & 83.1 \\
\hline Total & 193 & 170 & 88.1 \\
\hline
\end{tabular}

Fuente: elaboración propia según procesamiento de datos en SPSS.

En la tabla 1 se muestran los porcentajes alcanzados en cada ciclo, logrando un porcentaje a nivel general del $88.1 \%$ de los estudiantes asignados, además de un porcentaje no menor al $83.1 \%$ de los estudiantes asignados en cada ciclo de la carrera. La diferencia entre la columna de asignados y encuestados se debe a que algunos estudiantes no continuaron sus estudios dentro de la carrera sujeta de estudio. 
El instrumento utilizado fue el cuestionario, con una escala de Likert, por lo que fue necesario establecer su confiabilidad, el cual se determinó a través del coeficiente Alfa de Cronbach.

Tabla No. 2 Alfa de Cronbach

\begin{tabular}{lc}
\hline \multicolumn{2}{c}{ Estadística de fiabilidad } \\
\hline Alfa de Cronbach & Número de elementos \\
0.992 & 114 \\
\hline
\end{tabular}

Fuente: elaboración propia según procesamiento de datos en SPSS.

En consideración a la tabla anterior, el coeficiente de fiabilidad encontrado fue de 0.992, y de acuerdo a los baremos de interpretación utilizados, el instrumento se encuentra en nivel elevado de confiabilidad.

Dentro de la investigación se consideraron seis variables, de las cuales cuatro son competencias específicas, las cuales son funciones propias del profesor, tanto dentro como fuera del aula universitaria. A continuación, se describen.

Docencia universitaria: se entiende como la preparación e implementación por parte del profesor hacia el estudiante de estrategias de aprendizaje, métodos de formación, prácticas de enseñanza, evaluaciones de rendimiento estudiantil, planificación educativa, uso de TIC's, acompañamiento, motivación, dominio y adaptación del contexto en que se encuentre el profesor con sus estudiantes y así permita un máximo rendimiento académico logrando una mejor formación académica.

Investigación e innovación: es la aplicación de métodos, técnicas y procedimientos dirigidos para alcanzar soluciones a problemas en un área específica y así poder explicar, predecir e interpretar una problemática en un contexto dado, así también la innovación son las acciones encaminadas que el profesor realiza con sus estudiantes para analizar desde distintos puntos de vista un problema, además de aplicar recursos que mejoren el aprendizaje y la incorporación de nuevas ideas dentro de la temática del curso. 
Vinculación con la sociedad: es un proceso dinámico a través del cual el profesor universitario se proyecta para intervenir en la solución de los problemas de la comunidad en donde interactúa, y actúa en conjunto con otros compañeros profesores, estudiantes e instituciones privadas y/o estatales de diversos sectores, además de brindar acompañamiento durante todo el proyecto, la participación del profesor en estas actividades las realiza en la especialidad que posee, ya que forma parte de un equipo multidisciplinario realizando al final un informe en donde destaca todo el proceso y los resultados que se obtuvieron para su respectiva evaluación.

Gestión institucional: se entiende como la aplicación de procedimientos para el manejo de los recursos y desarrollo de las actividades de la institución enfocada en la mejora de investigación y calidad educativa considerando la planificación y participación del cuerpo de profesores con una adecuada distribución de trabajo acorde las capacidades del cuerpo de profesores que integran las diferentes carreras universitarias dentro de la institución.

Tabla No. 3 Resultados de competencias específicas

\begin{tabular}{ccccc}
\hline \multicolumn{5}{c}{ Competencias específicas } \\
\hline Niveles & $\begin{array}{c}\text { Docencia } \\
\text { universitaria }\end{array}$ & $\begin{array}{c}\text { Investigación e } \\
\text { innovación }\end{array}$ & $\begin{array}{c}\text { Vinculación con la } \\
\text { sociedad }\end{array}$ & $\begin{array}{c}\text { Gestión } \\
\text { institucional }\end{array}$ \\
\hline $\begin{array}{c}\text { No posee la } \\
\text { competencia }\end{array}$ & $2.9 \%$ & $7.6 \%$ & $1.8 \%$ & $5.9 \%$ \\
\hline Básico & $11.2 \%$ & $24.1 \%$ & $20.0 \%$ & $13.5 \%$ \\
\hline Intermedio & $35.9 \%$ & $35.3 \%$ & $16.5 \%$ & $21.8 \%$ \\
\hline Bueno & $33.5 \%$ & $21.2 \%$ & $33.5 \%$ & $33.5 \%$ \\
\hline Excelente & $16.5 \%$ & $11.8 \%$ & $28.2 \%$ & $25.3 \%$ \\
\hline Total & $100.0 \%$ & $100.0 \%$ & $100.0 \%$ & $100.0 \%$ \\
\hline
\end{tabular}

Fuente: elaboración propia según procesamiento de datos en SPSS.

De la tabla anterior se observa la variable docencia universitaria se encuentra en un nivel intermedio de logro con un $35.9 \%$, lo que significa que el profesor realiza su planificación didáctica y la comparte con sus estudiantes, establece los objetivos y estrategias, genera un buen ambiente de aprendizaje dentro del aula, promueve el autoaprendizaje y autonomía del estudiante, sin embargo, carece de recursos que acompañen al estudiante fuera del salón de clase y no establece con anticipación sus criterios de evaluación, además realiza actividades sin relevancia para los estudiantes. 
En cuanto a la variable de investigación e innovación se muestra que obtuvo un $35.3 \%$ lo que la ubica en el nivel intermedio de logro, quiere decir que el profesor identifica problemas o temas para investigar, se actualiza en sus contenidos de su curso, se involucra en equipos de investigación, pero debe de mejorar en sus técnicas y crear instrumentos propios para la recolección de información, además se refleja que no escribe ni publica artículos en revistas científicas.

Dentro de la variable de vinculación con la sociedad se tuvo un mejor resultado, ubicándose en el nivel bueno de logro con un 33.5\%, por lo que se afirma que el profesor participa de forma activa en los proyectos comunitarios que contribuyen a su desarrollo y les da seguimiento, busca apoyo de entidades públicas y privadas que colaboren con distintos recursos para determinado proyecto.

Por último, la variable de gestión institucional también se ubica en el nivel bueno de logro con un $33.5 \%$, de lo cual se afirma que el profesor es responsable con las tareas que le son asignadas dentro de la institución, hace uso eficaz de los recursos que se le proporcional, está comprometido con el crecimiento y promoción de la institución, pero debe de involucrarse de mejor manera en el seguimiento dentro de la evaluación de la carrera y en la resolución de conflictos dentro de sus equipos de trabajo.

Por otra parte, dentro de la investigación también se consideraron dos competencias transversales, las cuales son aquellas que forman parte en todo su trabajo como profesor universitario y se determina por sus valores, habilidades, destrezas personales que están dentro de su vida cotidiana. A continuación, se describen las variables que las conforman.

Valores y actitudes del ámbito personal: se entiende como la ética, respeto y compromiso del profesor hacia la institución, además de su manera de actuar con los estudiantes, su forma de ejercer la docencia, el apoyo y comprensión con sus colegas y estudiantes, así mismo el respeto hacia las distintas creencias étnicas, culturales y religiosas.

Valores y actitudes del ámbito interpersonal: es la actitud del profesor hacia el grupo de trabajo con el que convive, aplicación de estrategias que ayuden a crear ambientes propicios para generación de ideas, comunica- 
ción efectiva, valoración a la contribución y aportes de su equipo, ejercer liderazgo con todos sus compañeros. Los resultados de estas variables se muestran a continuación.

Tabla No. 4 Resultados de competencias transversales

\begin{tabular}{ccc}
\hline Niveles & $\begin{array}{c}\text { Competencias transversales } \\
\text { Valores } y \text { actitudes del ámbito } \\
\text { personal }\end{array}$ & $\begin{array}{c}\text { Valores y actitudes del ámbito } \\
\text { interpersonal }\end{array}$ \\
\hline $\begin{array}{c}\text { No posee la } \\
\text { competencia }\end{array}$ & $4.8 \%$ & $2.3 \%$ \\
\hline Básico & $7.6 \%$ & $8.3 \%$ \\
\hline Intermedio & $23.5 \%$ & $12.9 \%$ \\
\hline Bueno & $34.1 \%$ & $43.2 \%$ \\
\hline Excelente & $30.0 \%$ & $33.3 \%$ \\
\hline Total & $100.0 \%$ & $100.0 \%$ \\
\hline
\end{tabular}

Fuente: elaboración propia según procesamiento de datos en SPSS.

Según la tabla anterior, la variable de valores y actitudes del ámbito personal, el profesor se posicionó en el nivel bueno de logro con un $34.1 \%$ lo que indica que, el profesor actúa según los principios y valores de la Universidad y las normas del CUNZAC, disfruta su trabajo de ejercer docencias, es tolerante con sus estudiantes y afronta de buena manera los problemas y desafíos, además de que respeta las diferencias culturales y sociales de sus estudiantes y del resto de profesores.

Continuando con la variable de valores y actitudes del ámbito personal, nuevamente el profesor se ubica en el nivel bueno de logro con un $43.2 \%$, el cual es el porcentaje más alto alcanzado por el profesor en todas las competencias. Esto indica que el profesor es flexible a las exigencias de su grupo de trabajo, promueve una sana discusión de ideas y disciplina, presenta sus iniciativas con claridad, es líder y genera confianza, pero debe de aprender a comunicarse en el idioma inglés.

\section{Discusión}

Al realizar el análisis de resultados de las seis variables se observa que las competencias transversales obtuvieron mejores resultados que las competencias específicas, ya que ambos se ubicaron en el nivel bueno de lo- 
gro, siguiendo en el orden de niveles, las variables de vinculación con la sociedad y gestión institucional también se ubicaron en el mismo nivel, sin embargo, con porcentajes menores, y por último, las variables de docencia universitaria y de investigación e innovación resultaron con nivel intermedio, lo que refleja que los profesores, no cuentan con habilidades de investigación en temas de relevancia dentro de la carrera de Administración de Empresas, si no únicamente en investigación que forman parte de proyectos formativos dentro de la carrera.

En cuando al resultado obtenido en la variable de docencia universitaria se visualiza que el profesor no cuenta con un programa de acompañamiento al estudiante fuera del aula y que debe seguir capacitándose en metodologías de aprendizaje y fortalecer sus sistemas de evaluación formativa.

\section{Referencias}

Alvarado Rodas, H. R. (2020). Competencias digitales en el proceso de enseñanza-aprendizaje del docente y estudiante. Revista Guatemalteca De Educación Superior, 3(2), 12-23. https://doi.org/10.46954/revistages.v3i2.28

Arrieta Martínez, J. P. . (2020). La innovación del aprendizaje en el contexto universitario. Revista Académica CUNZAC, 3(1), 53-56. https://doi. org/10.46780/cunzac.v3i1.21

Aylett, R., \& Gregory, K. (1997). Criteria for teaching competence and teaching excellence in Higher Education. Londres: Falmer Press.

Flores Luin, W. P. M. (2018). Apreciación de los estudiantes sobre la didáctica de los docentes y su disposición al aprendizaje. Revista Guatemalteca De Educación Superior, 1(1), 27-33. https://doi.org/10.46954/ revistages.v1i1.16

Hernández, R., \& Mendoza, C. P. (2018). Metodología de la investigación. Las rutas cuantitativa, cualitativa y mixta. México: McGraw Hill Education. https://doi.org/10.17993/CcyLI.2018.15 
Julián Méndez, G. J. (2018). Competencia Intercultural de los estudiantes universitarios de la región Chortí del departamento de Chiquimula. Revista Académica CUNZAC, 1(1), 39-47. https://doi.org/10.46780/ cunzac.v1i1.4

Tobón, S. (2007). Gestión curricular y ciclos propedéuticos. Bogotá, Colombia: ECOE.

Torello, O. (2012). Las competencias del docente universitario: la percepción del alumno, de los expertos y del propio protagonista. REDU - Revista de Docencia Universitaria, 10(2), pp. 299-318. https://doi.org/10.4995/ redu.2012.6109

Torres, A. D., Badillo, M., Valentin, N. O., \& Ramírez, E. T. (2014). Las competencias docentes: el desafío de la educación superior. Innovación Educativa, 14(66), pp. 129-146.

Universidad de San Carlos de Guatemala. (2014). Plan académico de desarrollo estratégico gestión 2014-2018. Guatemala: USAC.

Universidad de San Carlos de Guatemala. (2015). Plan estratégico USAC2020. Guatemala: USAC.

Villarroel, V. A., \& Bruna, D. V. (2017). Competencias Pedagógias que caracterizan a un docente universitario de excelencia: un estudio de caso que incorpora la perspectiva de docentes y estudiantes. Formación Universitaria, 10(4), pp. 75-117. doi: 10.4067/S0718-50062017000400008.

Villela Cervantes, C. E. (2018). La educación virtual en las ciencias complejas. RevistaAcadémica CUNZAC, 1(1), 1-6. https://doi.org/10.46780/ cunzac.v1i1.1

Zuñiga, C. E. (2018). Percepción de los profesores del campus P. César Augusto Jerez García, S.J. de Quiché de la Universidad Rafael Landívar, acerca de sus competencias para la aplicación de estrategias de enseñanza y su utilidad para conseguir aprendizajes significativos en sus estudiantes, después del curso: estrategias de enseñanza para el aprendizaje significativo en el aula universitaria (Tesis de maestría). Universidad Rafael Landívar, Guatemala. 


\section{Sobre el autor \\ Marco Antonio Rosales Gómez}

Es Ingeniero Civil en el grado académico de licenciatura y maestro en ciencias graduado de la maestría en Docencia Universitaria con Orientación en Estrategias del Aprendizaje de la Universidad de San Carlos de Guatemala. Se ha desempeñado por 6 años como profesor universitario en el Centro Universitario de Zacapa, en las carreras de Ingeniería en Industrias Agropecuarias y Forestales y Administración de Empresas.

Copyright (c) Marco Antonio Rosales Gómez

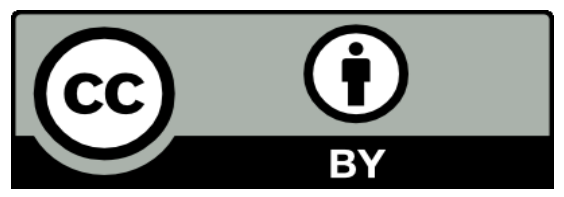

Este texto está protegido por una licencia CreativeCommons 4.0.

Usted es libre para compartir, copiar y redistribuir el material en cualquier medio o formato y adaptar el documento, remezclar, transformar y crear a partir del material para cualquier propósito, incluso comercialmente, siempre que cumpla la condición de atribución: usted debe reconocer el crédito de una obra de manera adecuada, proporcionar un enlace a la licencia, e indicar si se han realizado cambios. Puede hacerlo en cualquier forma razonable, pero no de forma tal que sugiera que tiene el apoyo del licenciante o lo recibe por el uso que hace. 